\title{
A Phase II Study of Glembatumumab Vedotin for Metastatic Uveal Melanoma
}

\author{
Merve Hasanov ${ }^{1,+}{ }^{+D}$, Matthew J. Rioth ${ }^{2,+}$, Kari Kendra ${ }^{3}$, Leonel Hernandez-Aya ${ }^{4}$, \\ Richard W. Joseph ${ }^{5}$, Stephen Williamson ${ }^{6}{ }^{D}$, Sunandana Chandra ${ }^{7}$, Keisuke Shirai ${ }^{8}$, \\ Christopher D. Turner ${ }^{9}$, Karl Lewis ${ }^{2}$, Elizabeth Crowley ${ }^{10}$, Jeffrey Moscow ${ }^{11}$, Brett Carter ${ }^{12}$ \\ and Sapna Patel ${ }^{1, *(D)}$
}

1 Department of Melanoma Medical Oncology, Division of Cancer Medicine, the University of Texas MD Anderson Cancer Center, Houston, TX 77030, USA; mhasanov@mdanderson.org

2 Division of Medical Oncology and Division of Biomedical Informatics and Personalized Medicine, Department of Medicine, University of Colorado Anschutz Medical Campus, Aurora, CO 80045, USA; Matthew.rioth@ucdenver.edu (M.J.R.); karl.lewis@ucdenver.edu (K.L.)

3 Division of Medical Oncology, Department of Medicine, the Ohio State University Comprehensive Cancer Center, Columbus, OH 43210, USA; Kari.Kendra@osumc.edu

4 Division of Medical Oncology, Department of Medicine, Washington University in St. Louis, St. Louis, MO 63110, USA.; lhernandezaya@wustl.edu

5 Department of Hematology and Oncology, Mayo Clinic Hospital, Florida, Jacksonville, FL 32224, USA; joseph.richard@mayo.edu

6 Division of Medical Oncology, Department of Medicine, University of Kansas Medical Center, Kansas City, KS 66160, USA; SWILLIAM@kumc.edu

7 Division of Hematology and Oncology, Department of Medicine, Northwestern University Feinberg School of Medicine, Chicago, IL 60611, USA; sunandana.chandra@northwestern.edu

8 Division of Hematology and Oncology, Department of Medicine, Norris Cotton Cancer Center, Dartmouth-Hitchcock Medical Center, Lebanon, NH 03766, USA; Keisuke.Shirai@hitchcock.org

9 Blueprint Medicines, Clinical Development, Cambridge, MA 02139, USA; cturner@blueprintmedicines.com

10 Celldex Therapeutics, Inc., Hampton, NJ 08827, USA; ecrowley@celldex.com

11 Cancer Therapy Evaluation Program National Institute of Health (CTEP), Bethesda, MD 20892, USA; jeffrey.moscow@nih.gov

12 Department of Thoracic Imaging, Division of Diagnostic Imaging, the University of Texas MD Anderson Cancer Center, Houston, TX 77030, USA; Bcarter2@mdanderson.org

* Correspondence: sppatel@mdanderson.org; Tel.: +1-713-792-2921; Fax: +1-713-745-1046

+ Hasanov and Rioth contributed equally to the manuscript.

Received: 7 July 2020; Accepted: 11 August 2020; Published: 13 August 2020

check for updates

\begin{abstract}
Glembatumumab vedotin (CDX-011, GV) is a fully human Immunoglobulin G2 monoclonal antibody directed against glycoprotein NMB coupled via a peptide linker to monomethyl auristatin E (MMAE), a potent cytotoxic microtubule inhibitor. This phase II study evaluated the overall response rate and safety of GV, glycoprotein NMB (GPNMB) expression, and survival in patients with metastatic uveal melanoma. Eligible patients with metastatic uveal melanoma who had not previously been treated with chemotherapy received GV $1.9 \mathrm{mg} / \mathrm{kg}$ every three weeks. The primary endpoint was the objective response rate (ORR). Secondary endpoints included GPNMB expression, progression-free survival (PFS), overall survival (OS), and toxicity analysis. GPNMB expression was assessed pre- and post-treatment via immunohistochemistry for patients with available tumor tissue. Out of 35 patients who received treatment, two patients had confirmed partial responses (PRs; 6\%), and 18 patients had a stable disease (SD; 51\%) as the best objective response. 38\% of the patients had stable disease $>100$ days. The grade 3 or 4 toxicities that occurred in two or more patients were neutropenia, rash, hyponatremia, and vomiting. The median progression-free survival was 3.1 months (95\% CI: 1.5-5.6), and the median overall survival was 11.9 months (95\% CI 9.0-16.9) in the evaluable study population. GV is well-tolerated in metastatic uveal melanoma. The disease
\end{abstract}


control rate was 57\% despite a low objective response rate. Exploratory immune correlation studies are underway to provide insight into target saturation, combination strategies, and antigen release.

Keywords: uveal melanoma; glembatumumab vedotin; phase II; clinical trial

\section{Significance}

Metastatic uveal melanoma is an orphan disease with no FDA-approved disease-specific treatment options. Immunotherapy and targeted therapy are not as effective as in cutaneous melanoma because of the differences in disease biology and clinical characteristics. This phase II trial of glembatumumab vedotin in patients with metastatic uveal melanoma showed a $57 \%$ disease control rate (complete response (CR), partial response (PR), and stable disease (SD)) despite a low objective response rate $(6 \%)$. Treatment was well-tolerated except for neutropenia, which was managed with granulocyte-macrophage colony-stimulating factor (GM-CSF).

\section{Background}

Uveal melanoma is the most common primary intraocular malignancy in adults and constitutes $3.1 \%$ of all melanoma diagnoses. In the United States, the incidence of uveal melanoma has remained stable at 5.1 per million for the past 50 years in contrast to the rising incidence of cutaneous melanoma [1,2]. Though treatment of the primary tumor is highly efficacious, approximately half of the 3000 patients who are diagnosed annually with uveal melanoma will develop metastatic disease [3]. Unfortunately, many of the therapeutic advances that have revolutionized the treatment of cutaneous melanoma have not effectively translated to the uveal melanoma population [4].

The disparity between cutaneous and uveal melanoma is likely attributable to differences in their biology. Whereas cutaneous melanoma is characterized by a UV-mediated high mutation burden and a high incidence of activating mutations in the BRAF protein, uveal melanoma carries a low mutational burden, no UV mutation signature, and a rare occurrence of BRAF mutations. Early canonical activating mutations in the MAPK pathway in GNAQ or GNA11 have not led to the successful targeting of the MAPK pathway [5,6]. Similarly, immunotherapies with checkpoint inhibitors have not demonstrated the same efficacy as in cutaneous melanoma [7]. In previously published work in metastatic uveal melanoma, PD-L1 expression in these tumors was found to be low-to-absent in the majority of cases, which may account for the low response rates to checkpoint blockades [8,9]. This is in contrast to the notable expression of other checkpoint molecules like indoleamine 2, 3-dioxygenase (IDO) and the T cell Ig and ITIM domain (TIGIT) [10]. Regrettably, clinical experience with these latter checkpoint modulators for uveal melanoma is not known. As such, uveal melanoma is left without a standard of care for metastatic disease, and this lack of effective treatment contributes to a median of approximately six-to-thirteen month overall survival rates for patients with metastatic uveal melanoma [11].

Glycoprotein NMB (GPNMB) is a transmembrane protein whose overexpression promotes the invasion and metastasis of cancer cells and is expressed at high levels in uveal melanoma. In preclinical studies, $86 \%$ of uveal melanoma specimens demonstrated positive immunohistochemistry (IHC) staining for GPNMB, though with variable intensity [12]. Glembatumumab vedotin (GV) is a fully humanized monoclonal antibody directed against GPNMB that is coupled to the potent cytotoxic microtubule inhibitor monomethyl auristatin E (MMAE) [13]. The proposed mechanism of action is that upon the binding of GPNMB, the complex is internalized, MMAE is released in the lysosome, and tumor cell death occurs as a result of microtubule-inhibition mediated apoptosis. In mouse xenografts with the GPNMB-expressing melanoma cell lines SK-Mel-2 and SK-Mel-5, treatment with GV was found to induce the complete regression of tumors [14-16].

Given these promising preclinical results and the biological plausibility of the anti-tumor mechanism, this phase II study was undertaken to determine the effect of single-agent GV on 
the overall response of patients with metastatic uveal melanoma while also determining the clinical safety, pharmacodynamics, and GPNMB expression of tumors.

\section{Methods}

\subsection{Study Design}

This single-arm, open label phase II trial assessed the efficacy of a single agent, GV, in patients with metastatic uveal melanoma who had not previously been treated with chemotherapy. Enrollment began in January of 2016 and concluded in September of 2017. Seven institutions participated in the trial. All eligible patients were treated with the investigational therapy after obtaining informed consent.

\subsection{Patient Selection}

Patients were considered eligible if they had a histologically or cytologically confirmed metastatic uveal melanoma. Patients with a previous history of uveal melanoma in whom the histologic or cytologic diagnosis was not possible were also allowed at the discretion of the treating investigator. Eligible patients had to be aged greater than 18 years, an Eastern Cooperative Oncology Group (ECOG) performance status of 2 or better, a life expectancy longer than three months, a measurable disease by RECIST version 1.1, adequate organ function, and treatment-naive for chemotherapy. Patients may have received radiation, immunotherapy, and liver-directed or targeted therapy, but all treatment must have been completed 28 days before study drug treatment and prior treatment-related toxicities had to be CTCAEv4 (Common Terminology Criteria for Adverse Events version 4) grade $\leq 1$. Both men and women and members of all races and ethnic groups were eligible. The protocol and amendments were approved by the Central Institutional Review Board for the National Cancer Institute under the study ID of 9855 . All participants of the study provided written informed consent before initiating study procedures.

\subsection{Dosing Regimen}

All eligible patients were intravenously treated with GV at a dose of $1.9 \mathrm{mg} / \mathrm{kg}$ actual body weight every three weeks as outpatients. GV was prepared by dilution in $5 \%$ dextrose and administered as a 90-min infusion without premedication. Patients who experienced grade 3 toxicity were treated with reduced doses of $1.3 \mathrm{mg} / \mathrm{kg}$ every three weeks and then, if recurrent, at $1 \mathrm{mg} / \mathrm{kg}$ every three weeks. An algorithm for the management of hypersensitivity or allergic reactions was provided to investigators.

\subsection{Response Criteria}

Investigator-determined tumor response was radiographically measured every six weeks from treatment initiation using RECIST version 1.1 [17].

\subsection{Toxicity Capture}

Clinical and laboratory assessments were conducted at baseline and every three weeks for up to 30 days following the off-treatment date. Adverse events were graded according to the National Cancer Institute (NCI) Common Terminology Criteria for Adverse Events version 4.0 (https://ctep.cancer.gov/protocolDevelopment/electronic_applications/ctc.htm\#ctc_40).

\subsection{Statistical Analysis}

The primary end point was the overall response rate. Secondary end points included overall survival, progression-free survival, clinical safety, tolerability, and pharmacodynamics changes in GPNMB expression by IHC. Patients who received at least one dose of therapy or who experienced objective disease progression during the first cycle of therapy were evaluable for the primary end point and the secondary end points. 
A single arm, non-randomized design using Simon's two stage design was used to evaluate the primary end point. In this design, 18 patients are enrolled in the first stage, and if at least one response is observed in this first stage, up to 14 additional patients are enrolled in the second stage. Assuming $15 \%$ of metastatic uveal melanoma samples do not express GPNMB and therefore would not be expected to respond, this study targeted an objective response rate (ORR) of $20 \%$ compared to the historical reference ORR of $5 \%$.

Toxicity was reported by type, frequency, and severity according to the NCI Common Toxicity Criteria v4.0. All patients who received any amount of study drug was evaluable for toxicity.

Progression-free survival (PFS) and overall survival (OS) survival curves were calculated using the Kaplan-Meier method. The median PFS and OS were reported with 95\% confidence intervals.

\subsection{Correlative Analysis}

Correlative analysis included changes in GPNMB expression via IHC after 1 cycle of GV and a correlation of development of skin rash in cycle 1 with response.

\subsection{Immunohistochemistry}

GPNMB expression was assessed via IHC at Mosaic Laboratories (Lake Forest, CA, USA). The GPNMB (goat polyclonal) IHC assay was designed and validated to be compatible with CLIA guidelines for "homebrew" class I test validation. The procedure for the IHC analysis of GPNMB (goat polyclonal) was performed using automated detection at room temperature (RT) on the Dako Link Autostainer 48. Specimens were sectioned at 3-5-micron thickness, mounted onto positive-charged glass slides, dried, baked, deparaffinized, and rehydrated. Tissue sections then underwent pretreatment using a FLEX Target Retrieval Solution at a high pH (1×, Dako, Catalog\# K8004 or S2367, Agilent, Santa Clara, CA, USA) for $40 \mathrm{~min}$ in the PT Link (Dako) set to $97{ }^{\circ} \mathrm{C}$. Slides were cooled to $65^{\circ} \mathrm{C}$ inside the PT Link, removed, and immediately placed into a FLEX Wash Buffer (Dako, Catalog\# K8007) for up to $5 \mathrm{~min}$ before placing onto the autostainer. Once the autostainer procedure was initiated, the slides were rinsed with a buffer immediately and after each of the following steps: (1) Incubate with anti-GPNMB antibody or isotype negative control for $30 \mathrm{~min}$; (2) detect with rabbit anti-goat (Vector Laboratories, Burlingame, CA, USA) for $15 \mathrm{~min}$ and PowerVision poly AP anti-rabbit IgG for $15 \mathrm{~min}$ (rinse for $5 \mathrm{~min}$ in a buffer); and (3) stain with a Warp Red buffer solution (BioCare Medical, Concord, CA, USA) for 7 min each. Upon the completion of the staining procedure, slides were counterstained with hematoxylin (Dako) for $2 \mathrm{~min}$ followed by a rinse in distilled water, a rinse with a wash buffer for $5 \mathrm{~min}$, and then another rinse in distilled water. Coverslip mounting occurred offline using an automated cover slipper in accordance with Mosaic Laboratories' standard operating procedures.

Staining was evaluated by a pathologist, and the evaluation of reactivity involved a combination of the following: the cellular localization of staining, staining intensity, subcellular localization, and the percentage of cells staining in the primary component of the tissue type of interest. The GPNMB (goat polyclonal) assay was evaluated on a semi-quantitative scale, and the percentage of cells staining at each of the following four levels was recorded as 0 (unstained), $1+$ (weak staining), 2+ (moderate staining), and $3+$ (strong staining). The interpretation was performed within the tumor cells of the entire tissue. All tumor cells were considered in the score. A total positive score (percent positive) was derived using variety of magnifications $(4 \times, 10 \times$, and $20 \times)$.

\section{Results}

\subsection{Demographics}

Between January 2016 and September 2017, 37 patients were enrolled in the study. The Early Drug Development Opportunity Program (EDDOP) sites were added in August 2016. EDDOP contributed four patients to this effort. Baseline demographics are presented in Table 1. The site of metastases was predominantly the liver, followed by the lung, the lymph node, deep soft tissue, bones, skin, 
and subcutaneous tissue. The seventh edition M-stage AJCC uveal melanoma - which designates M1a for tumors $3 \mathrm{~cm}$ or smaller, M1b for tumors $3-8 \mathrm{~cm}$, and M1c for tumors greater than $8 \mathrm{~cm}-$ was used. The enrolled population was balanced for M-stage.

Table 1. Baseline patient characteristics.

\begin{tabular}{|c|c|c|}
\hline Characteristics & No. of Patients & $\%$ \\
\hline \multicolumn{3}{|l|}{ Age in years } \\
\hline Median & 62 & \\
\hline Range & $29-79$ & \\
\hline \multicolumn{3}{|l|}{ Gender } \\
\hline Male & 18 & 51 \\
\hline Female & 17 & 49 \\
\hline \multicolumn{3}{|l|}{ Performance Status (ECOG) } \\
\hline 0 & 26 & 74 \\
\hline 1 & 9 & 26 \\
\hline \multicolumn{3}{|l|}{ M-Stage * } \\
\hline M1a & 16 & 46 \\
\hline M1b & 12 & 34 \\
\hline M1c & 7 & 20 \\
\hline \multicolumn{3}{|l|}{ Sites of Metastasis ** } \\
\hline Liver only & 17 & 48 \\
\hline Extrahepatic only & 3 & 9 \\
\hline $\begin{array}{c}\text { Both liver and extra- } \\
\text { Hepatic }\end{array}$ & 15 & 43 \\
\hline \multicolumn{3}{|l|}{ Extrahepatic sites } \\
\hline Lung & 14 & 40 \\
\hline Lymph node & 9 & 26 \\
\hline Bones & 6 & 17 \\
\hline Peritoneum/Soft tissue & 5 & 17 \\
\hline Subcutaneous & 2 & 14 \\
\hline Muscle & 1 each & 6 \\
\hline All other sites $* * *$ & & 3 \\
\hline
\end{tabular}

Abbreviation: ECOG, Eastern Cooperative Oncology Group. * 7th edition AJCC M-stage uveal melanoma: M1a for tumors $3 \mathrm{~cm}$ or smaller, M1b for 3-8 cm tumors, and M1c for tumors greater than $8 \mathrm{~cm}$. The enrolled population was balanced for M-stage. ** The most common site of metastasis was the liver. Patients could have more than one site of metastasis. ${ }^{* * *}$ Other extrahepatic sites were the brain, the orbit, the thyroid, the heart, the stomach, the spleen, the gallbladder, the adrenal gland, and the kidney.

\subsection{Efficacy}

Of 37 patients enrolled in the study, 35 were assigned to treatment with GV. One participant withdrew consent before starting the treatment, and another was ineligible to receive treatment due to elevated liver transaminases. There were two confirmed PRs (6\%) via RECIST 1.1 in patients with M1b and M1c disease in the liver, and there were no CRs. An SD was seen in 51\% $(n=18)$ as the best overall response in the study, while $40 \%$ of the patients experienced the progression of disease (PD) $(n=14)$. One patient did not have a post-baseline tumor assessment after being removed from study due to an adverse event in cycle 1 (grade 4 neutropenia). Another patient without a post-baseline tumor assessment died during cycle 1 due to PD and was counted in the PD rate. The disease control rate-defined as the sum of CR, PR, and SD-was 57\% $(n=20)$. Efficacy is presented in Figure 1 as a waterfall plot and in Table 2 as text. The median duration of response was 8.6 months (263 days with a range of 149-377 days). Three patients had new lesions appear at their first tumor assessments, thus qualifying these responses as the progression of disease despite reductions in target lesion(s). An SD lasting longer than 100 days was noted in 34\% $(n=12)$ of the total study population, representing the majority of patients with an SD. The median duration of the SD across the study was 4.8 (147 days) 
months. Figure 2 shows comparative computerized tomography images of metastatic uveal melanoma tumors before and in response to GV treatment.

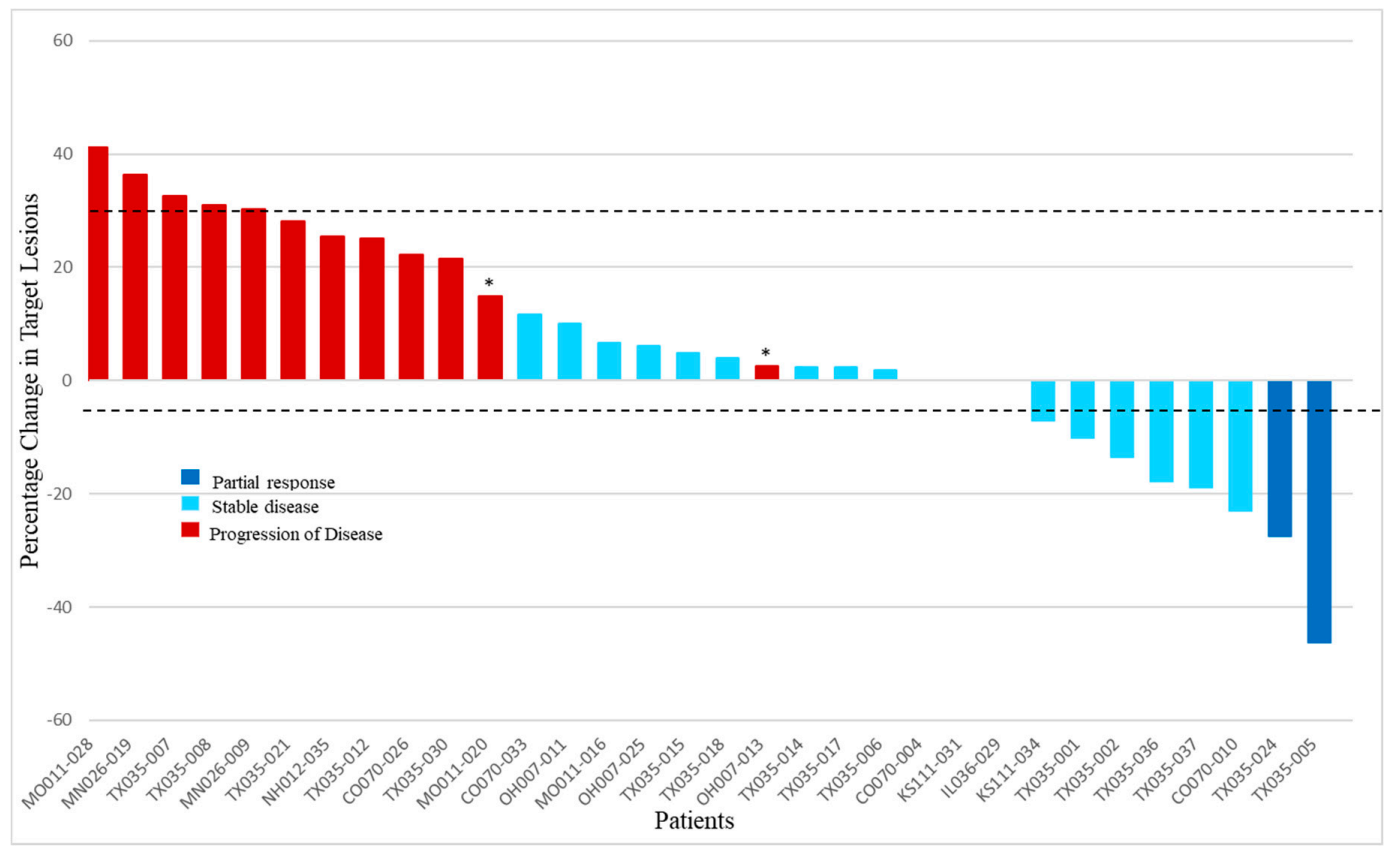

Figure 1. Best overall response of target lesions to glembatumumab vedotin. For each evaluable patient, the best response (defined by shrinkage in RECIST target lesions) is indicated ( $n=32$ ). Dark blue bars indicate the patients with partial response, light blue columns show the patients who had stable disease, and red columns represent patients with the progression of disease. Asterisks indicate the patients who had appearance of new lesions at the first tumor evaluation. Of the 3 patients not shown, one patient died during cycle 1 due to the clinical progression of disease without post-baseline tumor assessment, and another was removed for grade 4 neutropenia and acute kidney injury during cycle 1 and did not have post-baseline tumor assessment. The third patient had a complete disappearance of target lesion(s) at the first post-baseline tumor assessment but was noted to have new tumors elsewhere, so the best response was classified as the progression of disease. This patient did not have tumor measurements entered into the database and was therefore not included in the graphical output.

Table 2. Efficacy analyses.

\begin{tabular}{|c|c|c|}
\hline Response & No. & $\%$ \\
\hline Evaluable for response & \multicolumn{2}{|c|}{$(n=35)$} \\
\hline Complete Response (CR) & 0 & 0 \\
\hline Partial Response (PR) & 2 & 6 \\
\hline Stable Disease (SD) & 18 & 51 \\
\hline Disease Progression & 14 & 40 \\
\hline Unknown * & 1 & 3 \\
\hline Disease Control Rate $(C R+P R+S D)$ & 20 & 57 \\
\hline Stable Disease $>100$ days & 12 & 34 \\
\hline \multicolumn{3}{|l|}{ Duration of response, days } \\
\hline Median & \multicolumn{2}{|c|}{263} \\
\hline Range & \multicolumn{2}{|c|}{$149-377$} \\
\hline \multicolumn{3}{|l|}{ Duration of stable disease, days } \\
\hline Median & \multicolumn{2}{|c|}{147} \\
\hline Range & \multicolumn{2}{|c|}{$82-426$} \\
\hline
\end{tabular}

RECIST 1.1 criteria was used to determine tumor response. * This participant was removed from study after experiencing an adverse event in cycle 1 . There was no post-baseline tumor assessment. 

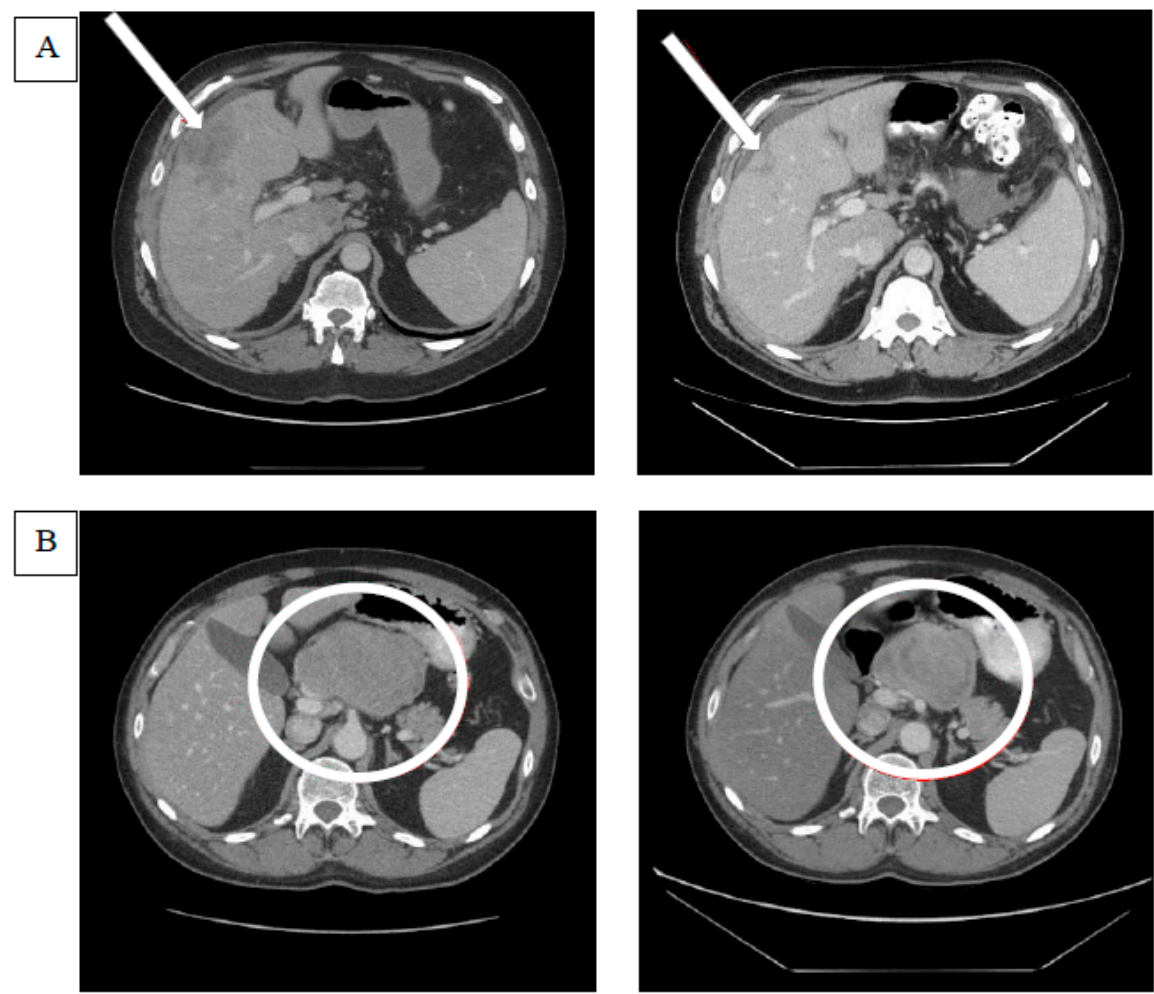

Figure 2. Examples of tumor responses after glembatumumab vedotin treatment. (A) The computerized tomography (CT) scan on the left indicates the baseline target lesion in the liver parenchyma that is pointed at by an arrow. The CT scan image on the right demonstrates the shrinkage in the target lesion after glembatumumab vedotin (GV). (B) The CT scan on the left demonstrates the target lesion in the porta hepatis lymph node, and the one on the right shows the changes in the lesion after GV. Though the lesion appears to have shrunk in size, the decrease in the lesion's short axis was not sufficient to meet the response criteria of RECIST 1.1. The target lesion response in the right panel was classified as a stable disease.

The median overall survival (Figure 3) for the evaluable population was 11.9 months (95\% CI: 9.0-16.9). The median progression-free survival was 3.1 months (95\% CI: 1.5-5.6). The range of OS was 0.5-40.2 months, whereas the PFS ranged from 0.5 to 30.5 months.
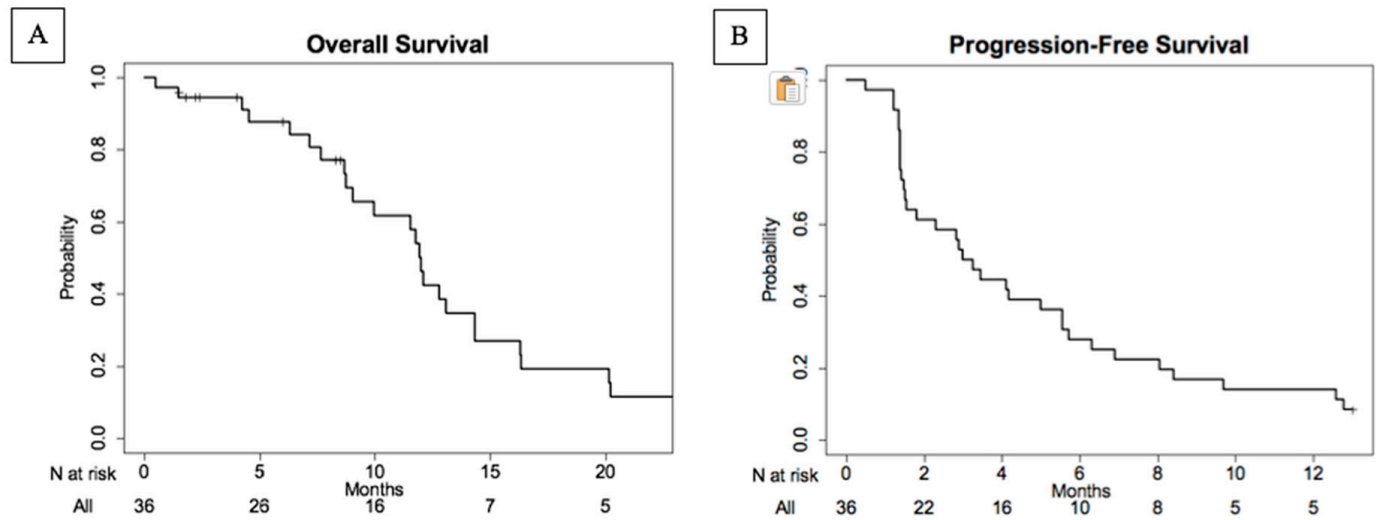

Figure 3. Kaplan-Meier plots of overall survival (A) and progression-free survival (B) in evaluable patient population. The median overall survival was 11.9 months (95\% CI: 9.0-16.9) ranging between 0.5 and 40.2 months. The median progression-free survival (PFS) was 3.1 months (95\% CI: 1.5-5.6) and ranged between 0.5 and 30.5 months. 


\subsection{Adverse Events}

All 35 patients who received GV were evaluable for toxicity. Common treatment-related adverse events (TRAEs) are presented as groups by organ class in CTCAE grades (Table 3). The most common TRAEs were skin and subcutaneous AEs, namely alopecia (80\%), maculopapular rash $(54 \%)$, and pruritus (51\%), as well as (less frequently) dry skin (9\%), skin hypopigmentation (9\%), and acneiform rash $(6 \%)$. Leukopenia $(69 \%)$, neutropenia $(60 \%)$, anemia $(40 \%)$, lymphopenia $(17 \%)$, and thrombocytopenia (14\%) were the most common hematologic AEs; meanwhile, elevated alanine aminotransferase/aspartate aminotransferase (ALT/AST) (63\%), nausea (51\%), diarrhea (31\%), elevated alkaline phosphatase $(26 \%)$, constipation $(23 \%)$, oral mucositis $(17 \%)$, and vomiting $(14 \%)$ were the most common gastrointestinal TRAEs. Other common TRAEs were fatigue $(57 \%)$, peripheral neuropathy $(43 \%)$, anorexia (37\%), and arthralgia (26\%). The most common grade $3 / 4 \mathrm{AE}$ was neutropenia $(48 \%)$, which was reversible and managed with the use of GM-CSF in subsequent cycles. Other grade $3 / 4$ AEs occurring in 1-2 (3-6\%) patients were leukopenia, elevated ALT/AST, nausea, diarrhea, constipation, vomiting, rash, fatigue, weight loss, hyponatremia, hypophosphatemia, and hypotension. There was only one grade 5 TRAE reported as encephalopathy, which resulted in death during the first treatment cycle. Upon further review of the data entry, this death was noted as disease progression in the liver with resultant hepatic encephalopathy, and it was not clearly related to GV. Twenty-seven serious adverse events (SAEs) were reported to the serious adverse event portal. Among these SAEs, three (9\%) events of neutropenia; two (6\%) events of hyponatremia, vomiting, and maculopapular rash; and one (3\%) event of elevated AST, diarrhea, hypotension, abdominal pain, nausea, constipation were reported as grade 3 SAEs. The reported grade 4 SAEs comprised seven (20\%) cases neutropenia, one (3\%) case of leukopenia, and one (3\%) case acute kidney injury. There was only one (3\%) grade $5 \mathrm{SAE}$ with encephalopathy, as detailed above.

Table 3. Treatment-related adverse events.

\begin{tabular}{|c|c|c|c|c|c|c|}
\hline \multirow[t]{2}{*}{ Adverse Event } & \multicolumn{2}{|c|}{ Any Severity } & \multicolumn{2}{|c|}{ CTCAE Grade 3} & \multicolumn{2}{|c|}{ CTCAE Grade 4} \\
\hline & No. & $\%$ & No. & $\%$ & No. & $\%$ \\
\hline Hematologic & & & & & & \\
\hline Leukopenia & 24 & 69 & 1 & 3 & & \\
\hline Neutropenia & 21 & 60 & 12 & 34 & 1 & 3 \\
\hline Anemia & 14 & 40 & & & 5 & 14 \\
\hline Lymphopenia & 6 & 17 & & & & \\
\hline Thrombocytopenia & 5 & 14 & & & & \\
\hline \multicolumn{7}{|l|}{ Gastrointestinal } \\
\hline Elevated ALT/AST & 22 & 63 & 1 & 3 & & \\
\hline Nausea & 18 & 51 & 1 & 3 & & \\
\hline Diarrhea & 11 & 31 & 1 & 3 & & \\
\hline Elevated ALP & 9 & 26 & & & & \\
\hline Constipation & 8 & 23 & 1 & 3 & & \\
\hline Mucositis, oral & 6 & 17 & & & & \\
\hline Vomiting & 5 & 14 & 2 & 6 & & \\
\hline Hyperbilirubinemia & 4 & 11 & & & & \\
\hline Abdominal pain & 2 & 6 & & & & \\
\hline Dry mouth & 2 & 6 & & & & \\
\hline Dyspepsia & 2 & 6 & & & & \\
\hline Oral pain & 2 & 6 & & & & \\
\hline
\end{tabular}


Table 3. Cont.

\begin{tabular}{|c|c|c|c|c|c|c|}
\hline \multirow[t]{2}{*}{ Adverse Event } & \multicolumn{2}{|c|}{ Any Severity } & \multicolumn{2}{|c|}{ CTCAE Grade 3} & \multicolumn{2}{|c|}{ CTCAE Grade 4} \\
\hline & No. & $\%$ & No. & $\%$ & No. & $\%$ \\
\hline \multicolumn{7}{|l|}{ Skin and Subcutaneous } \\
\hline Alopecia & 28 & 80 & & & & \\
\hline Rash, maculopapular & 19 & 54 & 2 & 6 & & \\
\hline Pruritus & 18 & 51 & & & & \\
\hline Dry skin & 3 & 9 & & & & \\
\hline Skin hypopigmentation & 3 & 9 & & & & \\
\hline Rash, acneiform & 2 & 6 & & & & \\
\hline \multicolumn{7}{|l|}{ General } \\
\hline Fatigue & 20 & 57 & 1 & 3 & & \\
\hline Pain & 6 & 17 & & & & \\
\hline Chills & 2 & 6 & & & & \\
\hline Flu like symptoms & 2 & 6 & & & & \\
\hline Localized edema & 2 & 6 & & & & \\
\hline Weight loss & 2 & 6 & 1 & 3 & & \\
\hline \multicolumn{7}{|l|}{ Nervous System } \\
\hline Peripheral Neuropathy & 15 & 43 & & & & \\
\hline Dysgeusia & 8 & 23 & & & & \\
\hline Headache & 3 & 9 & & & & \\
\hline Dizziness & 2 & 6 & & & & \\
\hline \multicolumn{7}{|l|}{ Musculoskeletal } \\
\hline Arthralgia & 9 & 26 & & & & \\
\hline Myalgia & 6 & 17 & & & & \\
\hline Pain in extremity & 3 & 9 & & & & \\
\hline Generalized muscle weakness & 2 & 6 & & & & \\
\hline \multicolumn{7}{|l|}{ Respiratory } \\
\hline Dyspnea & 5 & 14 & & & & \\
\hline \multicolumn{7}{|l|}{ Renal and Electrolytes } \\
\hline Hyponatremia & 5 & 14 & & & & \\
\hline Hypophosphatemia & 4 & 11 & 2 & 6 & & \\
\hline Hypokalemia & 3 & 9 & 1 & 3 & & \\
\hline Hyperkalemia & 2 & 6 & & & & \\
\hline \multicolumn{7}{|l|}{ Metabolism and Nutrition } \\
\hline Anorexia & 13 & 37 & & & & \\
\hline Hypoalbuminemia & 3 & 9 & & & & \\
\hline Hyperglycemia & 2 & 6 & & & & \\
\hline \multicolumn{7}{|l|}{ Vascular } \\
\hline Hot flashes & 3 & 9 & & & & \\
\hline Hypotension & 3 & 9 & 1 & 3 & & \\
\hline
\end{tabular}

Abbreviations: CTCAE: Common Terminology Criteria for Adverse Events, ALT: alanine aminotransferase, AST: aspartate aminotransferase, ALP: alkaline phosphatase. The treatment-related AEs had overall incidences $\geq 3 \%$, and all had grade 3-4 severity. Only one patient had a grade 5 treatment-related AE with encephalopathy. Empty data represent no reported toxicity.

Only one of the responders developed rash during the first cycle of the treatment, but it should be noted that rash occurring in cycle one did not correlate with response or PFS, as demonstrated in previous studies $[18,19]$.

\subsection{Gpnmb Tissue Expression}

Of the 32 patients, $26(81 \%)$ had tissues available for baseline, and $24(75 \%)$ had tissues available for GPNMB expression after one cycle of treatment. In baseline tissue, GPNMB was highly expressed across all metastatic tissues, with 11 (42\%) out of 26 available tissues demonstrating expression in $100 \%$ 
of tumor cells, whereas 8 (31\%) of 26 tissues exhibited expression in 20-95\% of tumor cells. Seven (27\%) of the baseline tissues did not demonstrate GPNMB expression. In the evaluation after one cycle of GV (Table S1), 8 (33\%) out of 24 available tissues demonstrated expression in $100 \%$ of tumor cells, and two $(8 \%)$ of them demonstrated zero expression in tumor cells, all unchanged from baseline. While nine (38\%) tumor samples showed paradoxical increases in GPNMB expression, three (13\%) had a decreased expression from baseline. Two patients ( $8 \%$ ) did not have a baseline tissue available to compare.

\section{Discussion}

This phase II trial of GV was conducted to evaluate its safety and efficacy in patients with metastatic uveal melanoma. The best objective response rate was $6 \%$, with no CR. However, there were $56 \%$ of patients with an SD, most of whom had an SD > 100 days. The median PFS was 3.1 months, and the median OS was 11.9 months. The side effect profile was tolerable with grade 3-4 neutropenia occurring in 56\% managed with GM-CSF.

The objective response rate to GV was not very improved compared to immunotherapy with PD-1 and PD-L1 antibodies (3.6\%) [7] or targeted therapy with selumetinib and dacarbazine (3\%) [20].

The median PFS in this study was higher than what has been seen in past studies with checkpoint blockade and targeted therapy $[5,7,20]$. A median OS of 11.9 months was likewise improved over past metastatic trials for this patient population. However, this is still a minimal improvement over historical cohorts and patients who only opt for the best supportive care [21].

The toxicities seen in this study have been previously reported in both cutaneous melanoma and triple-negative breast cancer [18,19]. Unlike those studies, the development of a rash in cycle 1 did not emerge as a biomarker for the response or clinical benefit. Elevated liver transaminases were likely higher in this study due to the common presence of hepatic metastases in uveal melanoma and, therefore, in our study population (91\%).

The baseline expression of GPNMB (73\%) appeared to be lower than the previous work of Williams et al. that demonstrated an 86\% expression of GPNMB in primary uveal melanoma tissues [12]. In breast and cutaneous melanoma, the expression of GPNMB is far lower, and GPNMB expression has been observed to be stable over time [19]. However, we observed a paradoxical increase in expression of GPNMB in 38\% of the tumor tissues after one cycle of GV treatment. This raises the possibility of inadequate target saturation by GV at a dose of $1.9 \mathrm{mg} / \mathrm{kg}$ in this disease population with a high percentage and intensity of GPNMB staining per tumor. Alternative mechanisms may be at play, thus leading to the upregulation of target.

The results of the study should be assessed within its limitations. First, this study had a small sample size. A current lack of standard treatment modalities for metastatic uveal melanoma makes it challenging to compare treatment efficacy. However, we compared the efficacy of GV to the best available efficacies amongst the clinical trials completed in the past. Though we had a high number of tumor tissue evaluated, the baseline and/or post-treatment tissue samples of some of the patients were not available for GPNMB expression.

\section{Conclusions}

In summary, GV was well-tolerated in the metastatic uveal melanoma patient population. The disease control rate was high and sustained despite a low objective response rate. Combination treatment strategies with immune checkpoint inhibitors are of interest, as are antibody-drug conjugates against other possible uveal melanoma targets.

Supplementary Materials: The following are available online at http://www.mdpi.com/2072-6694/12/8/2270/s1, Table S1: Pre and post treatment GPNMB expression.

Author Contributions: Conceptualization, S.P.; Data curation, M.J.R., K.K., L.H.-A., R.W.J., S.W., S.C., K.S., K.L., B.C., M.H. and S.P.; Formal analysis, S.P. and M.H.; Funding acquisition, C.D.T., E.C. and S.P.; Investigation, M.J.R., K.K., L.H.-A., R.W.J., S.W., S.C., K.S., K.L., J.M. and S.P.; Methodology, C.D.T., E.C., and S.P.; Resources, C.D.T., E.C., and S.P.; Supervision, S.P.; Writing-original draft preparation, M.H., M.J.R., and S.P.; Writing一review \& 
editing, M.H., M.J.R., K.K., L.H.-A., R.W.J., S.W., S.C., K.S., C.D.T., K.L., E.C., J.M., B.C. and S.P. All authors have read and agreed to the published version of the manuscript.

Funding: The study was supported by the Cancer Therapy Evaluation Program (CTEP) under NIH award number N01CM62202 and the NIH/NCI Cancer Center Support Grant under award number P30CA016672.

Conflicts of Interest: L.H.-A. received research funding from Celldex Therapeutics (Inst), Bristol Myers Squibb (Inst), Merck (Inst), Amgen (Inst), Roche (Inst), Regeneron (Inst), Novartis (Inst), Immunocore (Inst), Merck-EMD (Inst), Corvus (Inst), Polynoma (Inst), Genentech (Inst). S.W. received research funding from National Cancer Institute EDDOP Grant (Inst), Rogosin Institute (Inst), Bayer Health (Inst), Acceleron Pharma (Inst), EMD Serono (Inst), Nektar (Inst), Aleon Pharma (Inst), Novartis (Inst), Astellas Pharma (Inst), Regeneron (Inst), Pharmacyclics (Inst), Sanofi (Inst), Seattle Genetics (Inst), Bristol-Myers Squibb (Inst), Daiichi Sankyo (Inst), Sotio (Inst), Merck Serono (Inst). S.C. received honoraria from Bristol-Myers Squibb, Regeneron, Sanofi-Genzyme, Novartis, Exicure, EMD Serono. C.D.T. is employed by Blueprint Medicines. E.C. is employed by Celldex Therapeutics, Inc. J.M. is employed by CTEP. S.P. received honoraria from Castle Biosciences, Incyte, Cardinal Health, Merck \& Co., and research funding from Provectus (Inst), Ideaya (Inst), Bristol-Myers Squibb (Inst), Celgene (Inst), Novartis (Inst), GlaxoSmithKline (Inst), Deciphera (Inst), Reata Pharmaceuticals (Inst). All other authors declare no conflict of interest.

\section{References}

1. Singh, A.D.; Turell, M.E.; Topham, A.K. Uveal melanoma: Trends in incidence, treatment, and survival. Ophthalmology 2011, 118, 1881-1885. [CrossRef] [PubMed]

2. McLaughlin, C.C.; Wu, X.C.; Jemal, A.; Martin, H.J.; Roche, L.M.; Chen, V.W. Incidence of noncutaneous melanomas in the U.S. Cancer 2005, 103, 1000-1007. [CrossRef] [PubMed]

3. Diener-West, M.; Reynolds, S.M.; Agugliaro, D.J.; Caldwell, R.; Cumming, K.; Earle, J.D.; Hawkins, B.S.; Hayman, J.A.; Jaiyesimi, I.; Jampol, L.M.; et al. Development of metastatic disease after enrollment in the COMS trials for treatment of choroidal melanoma: Collaborative Ocular Melanoma Study Group Report No. 26. Arch. Ophthalmol. 2005, 123, 1639-1643. [CrossRef] [PubMed]

4. Yang, J.; Manson, D.K.; Marr, B.P.; Carvajal, R.D. Treatment of uveal melanoma: Where are we now? Ther. Adv. Med. Oncol. 2018, 10, 1-17. [CrossRef] [PubMed]

5. Luke, J.J.; Triozzi, P.L.; McKenna, K.C.; Van Meir, E.G.; Gershenwald, J.E.; Bastian, B.C.; Gutkind, J.S.; Bowcock, A.M.; Streicher, H.Z.; Patel, P.M.; et al. Biology of advanced uveal melanoma and next steps for clinical therapeutics. Pigment Cell Melanoma Res. 2015, 28, 135-147. [CrossRef]

6. Park, J.J.; Diefenbach, R.J.; Joshua, A.M.; Kefford, R.F.; Carlino, M.S.; Rizos, H. Oncogenic signaling in uveal melanoma. Pigment Cell Melanoma Res. 2018, 31, 661-672. [CrossRef]

7. Algazi, A.P.; Tsai, K.K.; Shoushtari, A.N.; Munhoz, R.R.; Eroglu, Z.; Piulats, J.M.; Ott, P.A.; Johnson, D.B.; Hwang, J.; Daud, A.I.; et al. Clinical outcomes in metastatic uveal melanoma treated with PD-1 and PD-L1 antibodies. Cancer 2016, 122, 3344-3353. [CrossRef]

8. Javed, A.; Arguello, D.; Johnston, C.; Gatalica, Z.; Terai, M.; Weight, R.M.; Orloff, M.; Mastrangelo, M.J.; Sato, T. PD-L1 expression in tumor metastasis is different between uveal melanoma and cutaneous melanoma. Immunotherapy 2017, 9, 1323-1330. [CrossRef]

9. Qin, Y.; Petaccia de Macedo, M.; Reuben, A.; Forget, M.A.; Haymaker, C.; Bernatchez, C.; Spencer, C.N.; Gopalakrishnan, V.; Reddy, S.; Cooper, Z.A.; et al. Parallel profiling of immune infiltrate subsets in uveal melanoma versus cutaneous melanoma unveils similarities and differences: A pilot study. Oncoimmunology 2017, 6, e1321187. [CrossRef]

10. Pan, H.; Lu, L.; Cui, J.; Yang, Y.; Wang, Z.; Fan, X. Immunological analyses reveal an immune subtype of uveal melanoma with a poor prognosis. Aging (Albany NY) 2020, 12, 1446-1464. [CrossRef]

11. Kuk, D.; Shoushtari, A.N.; Barker, C.A.; Panageas, K.S.; Munhoz, R.R.; Momtaz, P.; Ariyan, C.E.; Brady, M.S.; Coit, D.G.; Bogatch, K.; et al. Prognosis of Mucosal, Uveal, Acral, Nonacral Cutaneous, and Unknown Primary Melanoma From the Time of First Metastasis. Oncologist 2016, 21, 848-854. [CrossRef] [PubMed]

12. Williams, M.D.; Esmaeli, B.; Soheili, A.; Simantov, R.; Gombos, D.S.; Bedikian, A.Y.; Hwu, P. GPNMB expression in uveal melanoma: A potential for targeted therapy. Melanoma Res. 2010, 20, 184-190. [CrossRef] [PubMed]

13. Naumovski, L.; Junutula, J.R. Glembatumumab vedotin, a conjugate of an anti-glycoprotein non-metastatic melanoma protein $\mathrm{B} \mathrm{mAb}$ and monomethyl auristatin $\mathrm{E}$ for the treatment of melanoma and breast cancer. Curr. Opin. Mol. Ther. 2010, 12, 248-257. [PubMed] 
14. Pollack, V.A.; Alvarez, E.; Tse, K.F.; Torgov, M.Y.; Xie, S.; Shenoy, S.G.; MacDougall, J.R.; Arrol, S.; Zhong, H.; Gerwien, R.W.; et al. Treatment parameters modulating regression of human melanoma xenografts by an antibody-drug conjugate (CR011-vcMMAE) targeting GPNMB. Cancer Chemother. Pharmacol. 2007, 60, 423-435. [CrossRef]

15. Qian, X.; Mills, E.; Torgov, M.; LaRochelle, W.J.; Jeffers, M. Pharmacologically enhanced expression of GPNMB increases the sensitivity of melanoma cells to the CR011-vcMMAE antibody-drug conjugate. Mol. Oncol. 2008, 2, 81-93. [CrossRef]

16. Tse, K.F.; Jeffers, M.; Pollack, V.A.; McCabe, D.A.; Shadish, M.L.; Khramtsov, N.V.; Hackett, C.S.; Shenoy, S.G.; Kuang, B.; Boldog, F.L.; et al. CR011, a fully human monoclonal antibody-auristatin E conjugate, for the treatment of melanoma. Clin. Cancer Res. 2006, 12, 1373-1382. [CrossRef]

17. Eisenhauer, E.A.; Therasse, P.; Bogaerts, J.; Schwartz, L.H.; Sargent, D.; Ford, R.; Dancey, J.; Arbuck, S.; Gwyther, S.; Mooney, M.; et al. New response evaluation criteria in solid tumours: Revised RECIST guideline (version 1.1). Eur. J. Cancer 2009, 45, 228-247. [CrossRef]

18. Ott, P.A.; Hamid, O.; Pavlick, A.C.; Kluger, H.; Kim, K.B.; Boasberg, P.D.; Simantov, R.; Crowley, E.; Green, J.A.; Hawthorne, T.; et al. Phase I/II study of the antibody-drug conjugate glembatumumab vedotin in patients with advanced melanoma. J. Clin. Oncol. 2014, 32, 3659-3666. [CrossRef]

19. Yardley, D.A.; Weaver, R.; Melisko, M.E.; Saleh, M.N.; Arena, F.P.; Forero, A.; Cigler, T.; Stopeck, A.; Citrin, D.; Oliff, I.; et al. EMERGE: A Randomized Phase II Study of the Antibody-Drug Conjugate Glembatumumab Vedotin in Advanced Glycoprotein NMB-Expressing Breast Cancer. J. Clin. Oncol. 2015, 33, 1609-1619. [CrossRef]

20. Carvajal, R.D.; Piperno-Neumann, S.; Kapiteijn, E.; Chapman, P.B.; Frank, S.; Joshua, A.M.; Piulats, J.M.; Wolter, P.; Cocquyt, V.; Chmielowski, B.; et al. Selumetinib in Combination With Dacarbazine in Patients With Metastatic Uveal Melanoma: A Phase III, Multicenter, Randomized Trial (SUMIT). J. Clin. Oncol. 2018, 36, 1232-1239. [CrossRef]

21. Lane, A.M.; Kim, I.K.; Gragoudas, E.S. Survival Rates in Patients After Treatment for Metastasis From Uveal Melanoma. JAMA Ophthalmol. 2018, 136, 981-986. [CrossRef] [PubMed]

(C) 2020 by the authors. Licensee MDPI, Basel, Switzerland. This article is an open access article distributed under the terms and conditions of the Creative Commons Attribution (CC BY) license (http://creativecommons.org/licenses/by/4.0/). 